\title{
Primary umbilical endometriosis: To scope or not to scope?
}

\author{
A Chrysostomou, MD, FCOG (SA) MMed; S J Branch, MB BCh, FCOG (SA), MMed; N E Myamya, MB ChB, FCOG (SA) \\ Urogynaecology and Endoscopy Unit, Department of Obstetrics and Gynaecology, Faculty of Health Sciences, University of the Witwatersrand, \\ Johannesburg, South Africa
}

Corresponding author: A Chrysostomou (Andreas.Chrysostomou@wits.ac.za)

Background. Primary umbilical endometriosis (PUE) is a rare condition affecting $0.5-1 \%$ of all cases of extragenital endometriosis. The method of using routine laparoscopic inspection of the pelvis to exclude pelvic endometriosis has been applied extensively over the years. It has been demonstrated that even patients who have had no previous pelvic surgery or caesarean section, and have no symptoms of pelvic endometriosis or history of infertility, have presented with this condition.

Objective. To investigate whether patients with PUE should always undergo a laparoscopy to exclude pelvic endometriosis.

Methods. The study included women presenting with a history of painful umbilical nodules or bleeding from the umbilical nodule during or after menstruation in the absence of previous surgery either for gynecological disorders or caesarean section. The study began in January 2010 and ended in December 2016. All patients underwent umbilical biopsy confirming the presence of umbilical endometriosis before the diagnostic laparoscopy took place.

Results. Fourteen patients with cutaneous (scar) and umbilical endometriosis attended the clinic during the study period. Of these, only six cases $(42.8 \%)$ met the inclusion criteria of PUE clinically, and underwent diagnostic laparoscopy. Their mean age was 31.1 years (range 23 - 48), and the mean parity was 1.1 (range 0 - 3), with no history of previous pelvic surgery or caesarean section. Biopsies of the lesions confirmed the presence of endometrial tissue (gland and stroma) and haemorrhage. Diagnostic laparoscopy that took place immediately after the excision of umbilical endometriosis revealed no pelvic endometriosis.

Conclusion. A laparoscopy to exclude pelvic endometriosis should not be undertaken in patients who present with PUE, as there is a potential risk of introducing endometriosis into the pelvic cavity. Additionally, there is a risk of exposing the patient to unnecessary intervention and possible complications associated with the procedure.

S Afr J Obstet Gynaecol 2017;23(3):89-92. DOI:10.7196/SAJOG.2017.v23i2.1220

Endometriosis is defined by the presence of functional endometrial glands and stroma outside the endometrial cavity. It is a common gynaecological condition that affects up to $22 \%$ of all women ( $8-15 \%$ of women of reproductive age, and $6 \%$ of premenopausal women) ${ }^{[1,2]}$ The aetiology of endometriosis remains unclear; it is considered to be a 'disease of theories', with its pathophysiology only partially understood. Endometriosis usually occurs within the pelvic cavity. Common locations are the uterine wall, the fallopian tubes, the ovaries and the pelvic peritoneum. ${ }^{[3-5]}$ Pelvic endometriosis presents clinically with a triad of symptoms: pain (chronic pelvic pain and dysmenorrhea), menorrhagia and infertility.

Extrapelvic endometriosis affects up to $15 \%$ of patients, and can be found in almost all the organs (such as bowel, bladder, pericardium, pleura and even the brain). Extrapelvic endometriosis has varied signs and symptoms depending on the location. ${ }^{[6-8]}$

Cutaneous and umbilical endometriosis may occur in patients following gynaecological surgery or a caesarean section, owing to direct seeding after laparotomy or laparoscopy. ${ }^{[9]}$

The theory of lymphatic or vascular dissemination is favoured in the case of umbilical endometriosis with coexisting pelvic endometriosis. ${ }^{[10,11]}$

Primary umbilical endometriosis (PUE) is very rare, and not related to previous gynecological surgery or caesarean section. It is characterised by the presence of pigmented umbilical nodules associated with cyclical pain or bleeding during or immediately after menstruation. There is an estimated incidence of $0.5-1 \%$ of all cases of extragenital endometriosis. ${ }^{[12]}$

\section{Methods}

The study participants were women who presented with a history of painful umbilical nodules or bleeding from the umbilicus, during or immediately after menstruation, in the absence of previous surgery for either gynaecological disorders or a caesarean section. Patients with previous pelvic surgery or caesarean section who presented with scar or umbilical endometriosis were excluded from the study.

The study took place at a tertiary institution in South Africa, in the pelvic floor and endoscopy unit at the University of the Witwatersrand, between January 2010 and December 2016. A history was taken from all patients, followed by clinical examination. Vaginal ultrasound was performed in order to exclude any pelvic pathology. Abdominal ultrasound was performed to assess the umbilical masses.

Biopsies of the lesion were performed in all cases. Magnetic resonance imaging (MRI) was undertaken in cases where the umbilical mass was found to be fixed to the anterior abdominal wall during abdominal examination, or to be connected to the fascia during abdominal ultrasound. MRI was performed in order to assess the depth of the lesion, specifically in relation to the fascia and peritoneum. 
All cases with confirmed primary umbilical endometriosis were admitted for a wide excision of the lesion, as well as diagnostic laparoscopy for the assessment of pelvic endometriosis.

\section{Results}

Of the 14 cases with cutaneous (scar) and umbilical endometriosis that were referred to the pelvic floor and endoscopy unit during the study period, only 6 (42.8\%) fulfilled the criteria of primary umbilical endometriosis and were included in the study.

The mean age of the patients included in the study was 31.1 years. Patient characteristics are shown in Table 1. All six patients presented with having had a dark brown nodule on the umbilicus for a period of 6 months - 4 years (mean 18 months), associated with cyclical pain during or immediately after menstruation. Three of the cases reported cyclical pain that was associated with bloody discharge from the umbilicus during menstruation. Histories revealed no previous pelvic surgery or caesarean sections, and no abdominal pain (dysmenorrhoea or secondary dyspareunia). Infertility was not given as a reason for presentation. Per vaginal examination and transvaginal ultrasound revealed no gynaecological pathology or presence of endometriosis.

Abdominal ultrasound revealed the presence of a hypoechoic superficial mass in the umbilicus for four out of the six cases. Two of the cases with PUE underwent MRI to determine the depth of the lesion in relation to the fascia, as the lesion, on abdominal palpation, was found to be fixed. Biopsies of the umbilical mass were performed for all cases. Histology revealed the presence of functional endometrial tissue (gland and stroma) and associated haemorrhage.

All cases underwent wide excision of the lesion under general anaesthesia, followed by laparoscopy that served to confirm that PUE was not associated with pelvic endometriosis.

The follow-up period ranged from 6 months (since the last case entered the study) to 6 years (since the first case entered the study), with a mean follow-up period of 36 months.

\section{Discussion}

PUE is a term used to describe the presence of functional endometrial glands and stroma in the umbilicus, in the absence of previous surgeries (for either gynaecological disorders or caesarean sections). In cases with previous surgery, umbilical endometriosis should be defined as secondary umbilical endometriosis, which is more common than PUE, and is most likely due to iatrogenic direct seeding of the endometrial cells, either during laparoscopic or open surgical procedures. ${ }^{[9]}$ The theory of lymphatic or vascular dissemination is favoured in the case of umbilical endometriosis with coexisting pelvic endometriosis. ${ }^{[10,11]}$ The etiology of PUE remains unclear; however, several etiological theories have been proposed: these include coelomic metaplasia of the urachus

\section{Table 1. Patient demographics}

\begin{tabular}{ll}
\hline Age (years), range (mean) & $23-48(31.1)$ \\
Parity, range (mean) & $0-3(1)$ \\
Race, $n(\%)$ & \\
$\quad$ White & 0 \\
$\quad$ Indian & 0 \\
$\quad$ Coloured, & $1(16.6)$ \\
$\quad$ Black & $5(83.3)$ \\
Duration of symptoms (months), range (mean) & $6-48(18)$
\end{tabular}

remnant; direct extension through the round ligament; or congenital presence of developmentally displaced endometrial tissue.

PUE is rare, and not related to previous gynaecological surgery or caesarean section, with an estimated incidence of $0.5-1 \%$ of all cases of extragenital endometriosis. ${ }^{[12]}$ It is characterised by the presence of a pigmented umbilical nodule of variable size, from a few millimetres to a few centimetres, that is associated with cyclical pain or bleeding during or immediately after menstruation (Fig. 1).

The mean age of presentation in our study being 31.1 years, with the youngest patient 23 years old, was in keeping with the review performed by Victory et al.. ${ }^{[13]}$ who also found that the youngest patient in their series was 23 years old.

Endometriosis involving the abdominal wall is termed cutaneous endometriosis, and is commonly associated with surgical scars. ${ }^{[14]}$ Two cases with cutaneous (scar) endometriosis were found during the study period, both showing good progress following excision of the lesion. Six out of 12 cases with histologically proven umbilical endometriosis were found to have a previous history of caesarean section and pelvic surgery, and were therefore excluded from the study. The six patients with PUE who were included in this study had no clinical history suggestive of pelvic endometriosis, and no infertility issues.

Cyclical pain with a palpable mass is the most commonly presenting symptom of this condition. In our study, all patients had these classical cyclical symptoms. In spite of this, the diagnosis was delayed by an average of 18 months (range 6 - 48 months). This delay in diagnosis can be explained by the fact that our patients visited several doctors, both general practitioners and specialists, in local clinics and the private sector, before proceeding to biopsy of the lesion in a tertiary institution such as ours.

Histology is the mainstay of the diagnosis of umbilical endometriosis, as it is important to exclude other pathologies such as malignant melanoma, the 'Sister Joseph nodule' - a manifestation of intra-abdominal malignancy - or other dermatological conditions.

MRI is the most accurate method used to determine the depth of the lesion, specifically in relation to the sheath and the peritoneum. This is an important step when the excision of the umbilical nodule is undertaken. Two out of the six cases with PUE underwent MRI, which revealed that the umbilical nodule was penetrating the fascia (Fig. 2).

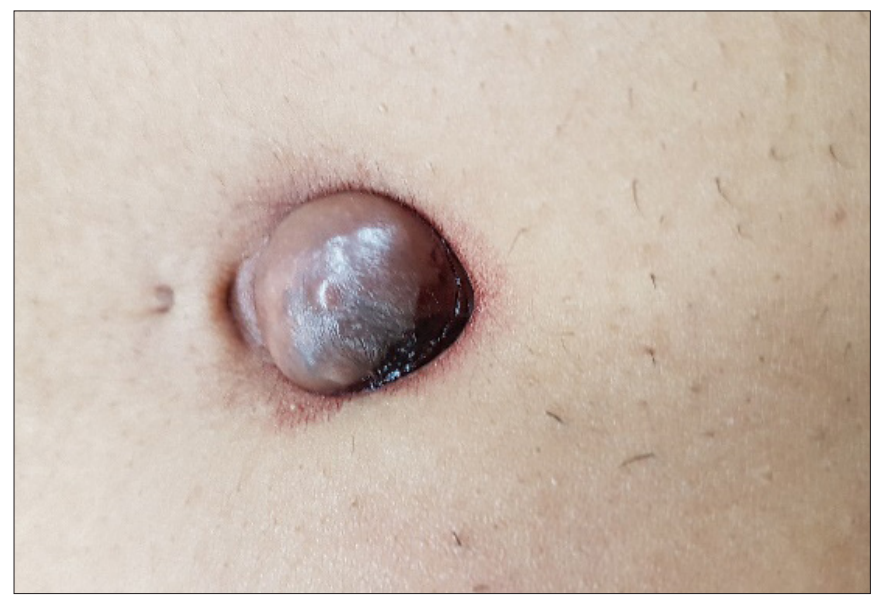

Fig. 1. Physical examination revealed the presence of a brown, moderately painful nodule of about $2 \mathrm{~cm}$ in diameter located deep in the umbilical fold, with signs of recent bleeding. 
There is no standard management regime for umbilical endometriosis, as there is a limited number of cases. Medical management using progesterone, danazol, norethisterone and gonadotropin-releasing hormone analogues has not shown reliable results. Nonetheless, some authors have reported success in relieving the symptoms and reducing the size of the endometrial nodule using medical hormonal treatment. ${ }^{[2,14]}$

All the cases in this study underwent total removal of the umbilicus. The surgical technique used was total excision of the endometriosis lesion, obtaining an adequate rim of normal tissue all around in order to avoid local recurrence (Fig. 3). Two cases underwent repair of the underlying fascia and peritoneum. Our study is comparable to others ${ }^{[1,4-16]}$ that have show that total excision of the lesion is associated with no recurrence during follow-up. The mean follow-up period for our cases was 36 months. During the follow-up period, there were no signs or symptoms of local recurrence. All cases included in the study underwent diagnostic laparoscopy. No pelvic endometriosis was found during diagnostic

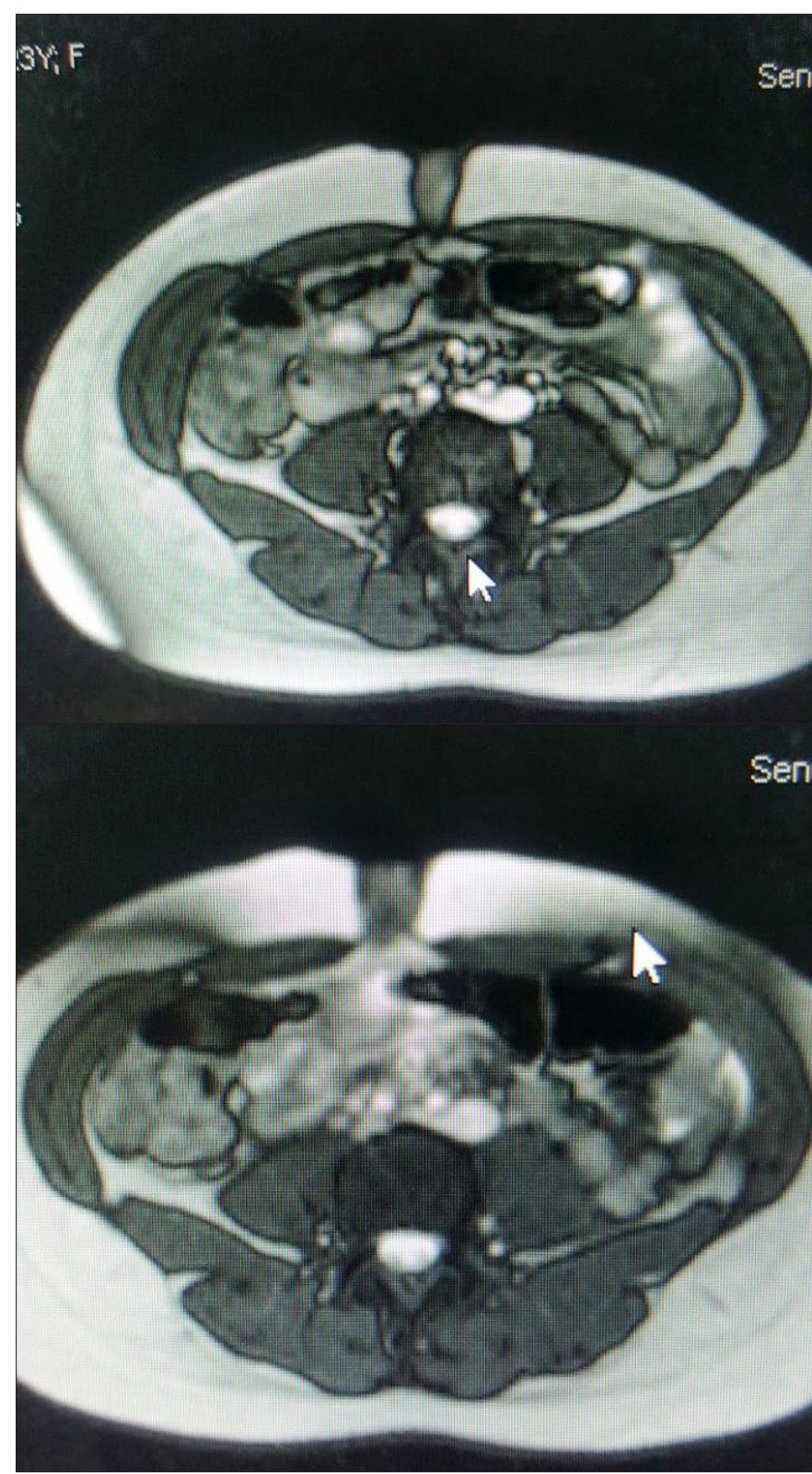

Fig. 2. Invasion of the umbilical endometriosis through the fascia sheet involving the peritoneum. laparoscopy. This supports the findings of others who have suggested that there is no need for laparoscopic assessment in cases of PUE. ${ }^{[4]}$

\section{Conclusion}

Umbilical endometriosis is an increasingly diagnosed and reported condition. The primary physician should be aware of this condition, to help in early diagnosis and treatment. In patients with PUE in which pelvic symptoms and signs are absent, laparoscopy to exclude pelvic endometriosis should not be undertaken. There is a potential risk of introducing endometriosis into the pelvic cavity, as well as a risk of exposing the patient to prolonged operating time, unnecessary

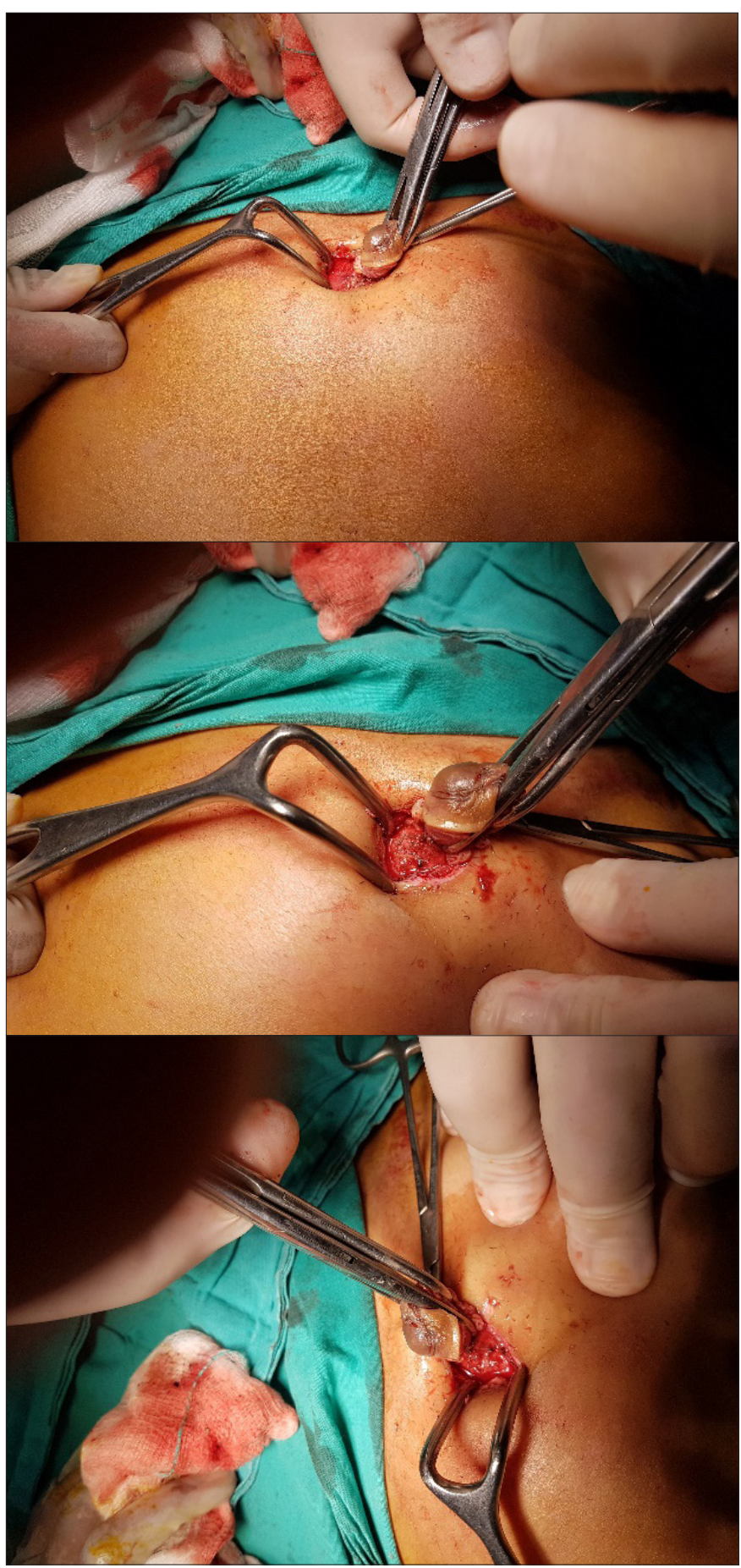

Fig. 3. Surgical removal of the umbilical nodule. The lesion was entirely excised down to the sheath, together with a $0.5 \mathrm{~cm}$ circumferential rim of macroscopically normal skin. 
interventions and potential complications. Complete excision prevents recurrence and should be offered even if this results in fascial defects requiring primary closure.

\section{Acknowledgements. None}

Author contributions. Data collection and write-up: AC; editing: SJB; assisting in theatre: NEM.

Funding. None.

Conflicts of interest. None.

1. Spaziani E, Picchio M, Di Philippo A, De Cristofano C, Ceci F, Stagnitti F. Spontaneous umbilica endometriosis: A case report with one year follow-up. Clin Exp Obstet Gynecol 2009;36(4):263-264. 2. Pugliarellos R, Colato C, Girolomoni G. Endometriosis of umbilical cicatrix: Case report and review of the literature. Acta Dermatovenerol Croat 2000:16:218-221.

3. Catalina-Fernandez I, Lopez-Presa D, Saenz-Santamaria J. Fine needle aspiration cytology in cutaneous and subcutaneous endometriosis. Acta Cytol 2007;51(3):380-384.

4. Argawal A, Fond YF. Cutaneous endometriosis. Singapore Med J 2008;49(9):704-709.

5. Pramanik SR, Mondal S. Primary umbilical endometriosis: A rarity. J Hum Reprod Science 2014;7(4):269-271. https://doi.org/10.4103\%2F0974-1208.147495

6. Kaushik R, Gulati A. Inguinal endometriosis: A case report. J Cytol 2008;25(2):73-74. https://doi org/10.4103/0970-9371.42455
7. Wolf G, Singh K. Caesarean scar endometriosis: A review. Obstet Gynecol Surv 1989;44(2):89-95. https://doi.org/10.1097/00006254-198902000-00003

8. Medeiros FC, Cavalcante DI, Medeiros MA, Eleuterio J. Fine needle aspiration cytology of scar endometriosis: Study of seven cases and literature review. Diagn Cytopathol 2011;39(1):18-21. https://doi.org/10.1002/dc.21319

9. Konincxk PR, Donders G, ven Decruys H. Umbilical endometriosis after unprotected removal o uterine pieces through the umbilicus. J Am Assoc Gynecol Laparosc 2000;7(2):727-732. https://doi. org/10.1016/s1074-3804(00)80045-6

10. Friedman PM, Rico MJ. Cutaneous endometriosis. Dermatol Online J 2000;6(1):8.

11. Igawa HH, Ohura T, Sugihara T, Hosokawa M, Kawamura K, Kaneko Y. Umbilical endometriosis. Ann Plast Surg 1992;29(3):266-268. https://doi.org/10.1097/00000637-199209000-00014

12. Freschknecht F, Rao L, Fleischmann A, Dreher E, Luscher KP, Mueller MD. Umbilical endometriosis. Surg Endosc 2001;18(2):347.

13. Victory R, Diamond MP, Johns DA. Villar's nodule: A case report and systematic review of endometriosis externa of the umbilicus. J Minim Invasive Gynecol 2007;14(1):23-32. https://doi org/10.1016/j.jmig.2006.07.014

14. Tech WT, Vollenhoven B, Harris PI. Umbilical endometriosis, a pathology that a gynecologis may encounter when inserting the Veres needle. Fertil Steril 2006;86(6):1764el-2. https://doi org/10.1016/j.fertnstert.2006.03.070

15. Bagade PV, Giurguis MM. Menstruating from the umbilicus as a rare case of primary umbilical endometriosis: A case report. J Med Case Rep 2009;3:9326. https://doi.org/10.1186/1752-1947-39326

16. Dadhwal V, Gupta B, Dasgupta C, Shende U, Deka D. Primary umbilical endometriosis: A rare entity. Arch Gynecol Obstet 2011;283(Suppl 1):S119-S120. https://doi.org/10.1007/s00404-010-1809-2

Accepted 29 November 2017. 